\title{
Marriage and Psychological Wellbeing: The Role of Social Support
}

\author{
Laura K. Soulsby, Kate M. Bennett \\ Department of Psychological Sciences, University of Liverpool, Liverpool, UK \\ Email: I.k.soulsby@liv.ac.uk
}

Received 29 April 2015; accepted 21 August 2015; published 25 August 2015

Copyright (C) 2015 by authors and Scientific Research Publishing Inc.

This work is licensed under the Creative Commons Attribution International License (CC BY). http://creativecommons.org/licenses/by/4.0/

c) (i) Open Access

\section{Abstract}

The married consistently report better levels of psychological health compared to the unmarried. Using a cross-sectional questionnaire design, this research examines to what extent this relationship between marital status and psychological wellbeing can be explained by perceived social support. The data reveal that, after controlling for demographic variables, number of daily hassles and coping strategies, widowed and divorced adults report significantly poorer psychological health compared to those who remain married. Moreover, while there was limited evidence that perceived social support moderates the association between marital status and psychological wellbeing, perceived social support did emerge as a significant mediator of this relationship. Perceived social support explained the influence of being widowed, divorced and never married on psychological wellbeing, such that lower levels of support in these groups resulted in poorer psychological health. Thus, social support may be an important variable for interventions to minimize the negative consequences of a transition out of marriage.

\section{Keywords}

Psychological Health, Social Support, Marriage, Divorce, Widowhood

\section{Introduction}

Researchers have yet to fully understand how or why marriage protects people from poor health and the relationships between marital status, social support and psychological wellbeing are not well understood. Previous research has acknowledged the associations between marital status and psychological wellbeing (e.g. Waite, 1995); social support and psychological wellbeing (e.g. Thoits, 1995); and marital status and social support (e.g. Pugliesi \& Shook, 1998). Yet, to date, limited research attention has been paid to the inter-relations between marital status, social support and psychological wellbeing, or the role of social support in the association between 
marital status and psychological health (Bierman, Fazio, \& Milkie, 2006; Hewitt, Turrell, \& Giskes, 2010). Sherbourne and Hays (1990), for example, found an indirect effect of marital status on psychological health through social support, but the findings were drawn from a chronically ill sample and may not be a reflection of the wider population. In a longitudinal study investigating marital loss, Hewitt et al. (2010) found that social support mediated the relationship between marital loss and psychological health, and that for widowed men, social support moderated psychological health. This research aims to build on the limited existing evidence. It makes use of a broader range of marital status groups and three measures of psychological wellbeing to assess the role of social support in the relationship between marital status and psychological health.

Social support can be described as the emotional, instrumental or tangible aid exchanged between members of the social network (e.g. House, Landis, \& Umberson, 1988). A large body of research demonstrates that social support is related to psychological health (House, Landis, \& Umberson, 1988). Two pathways by which social support may be associated with psychological wellbeing have been outlined in the literature (Cohen \& Wills, 1985). First, perceived social support may be associated with psychological wellbeing because it provides a buffer and, therefore, protects against the negative effects of stressful events. In this view, at times of high stress individuals with high levels of social support, in contrast to individuals with low levels of social support, have better psychological wellbeing. For example, research on spousal bereavement demonstrates that social support influences the coping process and buffers the effects of stressors on health (Walker, MacBride, \& Vachon, 1977). Alternatively, social support may directly influence psychological wellbeing independently of any external stressor where those with higher levels of social support experience better psychological wellbeing and those with lower levels of social support experience poorer psychological wellbeing, regardless of levels of stress.

A large literature documents the association between marital status and psychological health, with the married consistently found to enjoy better health compared to their unmarried counterparts (Gove, Hughes, \& Style, 1983; Hewitt et al., 2010; Waite \& Gallagher, 2000; Wyke \& Ford, 1992). Divorce and widowhood are still widely accepted as difficult transitions with important negative implications for psychological health, and formerly married people report lower levels of psychological health than never married people (Gove et al., 1983; Marks, 1996; Overbeek et al., 2006). However, while the psychological benefits of marriage are well documented, conclusions are often challenged by the question of causality and, to date, few studies have considered the role of social support in this relationship. Coyne and DeLongis (1986) suggested that the support provided by a spouse is not easily obtained elsewhere. Although a variety of explanations have been offered for marital status differences in psychological health, most can be categorized as describing either selection effects, suggesting that healthy individuals are selected into marriage and unhealthy ones are selected out (e.g. Stutzer \& Frey, 2006); or protection (e.g. Waite, 1995) and causation (e.g. Pearlin \& Johnson, 1977) effects, which suggest that marital status affects health as a consequence of between-group differences in access to resources, including social support. Specifically, marital protection would suggest that it is the presence of support in a marriage that facilitates better psychological health, while causation suggests it is the loss of previous support that results in lower levels of psychological wellbeing in the formerly married.

Marital status appears to be related to access to social support (e.g. Duffy, 1993; Ross, 1995). However, McLaughlin, Horwitz and White (2002) suggested that it is the presence of a supportive partner rather than marital status per se that best accounts for psychological wellbeing. Dush and Amato (2005) considered relationship status in terms of level of commitment and found that married individuals reported the highest level of subjective wellbeing, followed by cohabiting individuals, individuals dating one person in a steady relationship, followed by individuals dating multiple people, and finally, individuals not dating. Similar results have been found by Ross (1995) and Soons and Liefbroer (2008). The current study, therefore, includes people who are married, widowed, divorced, cohabiting, never married, those in a relationship but living apart (LAT), and those who have remarried.

This study aims to investigate the interrelationship between marital status, psychological wellbeing and perceived social support in order to achieve a clearer understanding of the causal relationships. A central question in this study is to what extent can marital status differences in psychological wellbeing be explained by differences in perceived social support? One effective method of assessing the role of support in the marital statuspsychological wellbeing relationship is to use moderation and mediation analyses, which allow for the estimation of direct and indirect effects of social support on psychological wellbeing in this cross-sectional data set. Moderation analyses test the buffering hypothesis to see if the direction and/or strength of the causal relationship between marital status and psychological wellbeing vary depending on the level of perceived social support. 
Mediation analyses test the direct effect of perceived social support on psychological wellbeing and evidence of mediation would suggest that marital status would have an indirect effect on psychological wellbeing through perceived social support (Baron \& Kenny, 1986).

\section{Data Collection}

\subsection{Sampling}

The study sample included a total of 510 British adults, with 375 women and 135 men completing the questionnaire from a range of ages, marital statuses and social backgrounds. The impact of self-selection effects was minimized by using multiple sites to host the survey link with the view that different sites would be likely to attract different types of participants. Multiple submissions have been found to be rare in internet experiments (Krantz \& Dalal, 2000; Reips, 2002). However, they were controlled by checking personal identifying items, and time and date consistency of responses, as recommended by Schmidt (1997). The median sample age was 32 years, with a range from 18 to 91 years. All participants provided informed consent and the study was approved by the University of Liverpool Ethics committee.

\subsection{Measures}

Dependent variable. Three measures of psychological wellbeing are explored in this paper: General Health Questionnaire (GHQ12; Goldberg, 1978); Center for Epidemiologic Studies Depression scale (CES-D; Radloff, 1977); and Satisfaction with Life Scale (SWLS; Diener, Emmons, Larsen, \& Griffin, 1985). GHQ12 measured respondents' assessment of their present state relative to their usual state in terms of feelings of happiness, ability to cope, sleep disruption, confidence, and depression on a 4-point Likert response scale. Scores were coded using Likert coding 0 to 3 to generate a total score from 0 to 36, with a higher score being indicative of poorer psychological wellbeing. CES-D consisted of 20 items that consider mood, somatic complaints, social interaction and motor functioning, scored on a 4-point Likert-scale. Likert coding 0 to 3 generated a scoring range from 0 to 60, with higher scores indicating higher levels of depressive symptoms. SWLS was used as a global measure of life satisfaction, assessing a cognitive rather than affective component of wellbeing (Pavot \& Diener, 1993). Items were coded on a 7-point Likert response scale and scores ranged from 5 (low satisfaction) to 35 (high satisfaction).

Independent variables. Marital status ${ }^{1}$ had 7 levels (married; widowed; divorced; cohabiting; never married; Living Apart Together (LAT); and remarried). Dummy coding $(0,1)$ was applied, with the married group used as the reference category as it is considered the normative status (Cohen \& Cohen, 1983). Social support was measured by the Medical Outcomes Study Social Support Survey (MOS-SSS; Sherbourne \& Stewart, 1991), a 19-item self-report scale used to assess perceived social support from family, friends and a significant other. The 5-point Likert response scale gave a continuous overall functional social support index with values ranging from 0 to 95 where a higher score indicates higher levels of perceived support.

Control variables. Daily hassles (Survey of Recent Life Experiences (SRLE; Kohn \& Macdonald, 1992), coping strategies (Brief COPE; Carver, 1997) and demographic variables were included as controls. Higher scores on the 41-item SRLE represent a higher number of daily hassles, with an overall scoring range from 41 to 164. Brief COPE comprised of 14 two-item coping subscales including active coping, planning, positive reframing, acceptance, humor, religion, using emotional support, using instrumental support, self-distraction, denial, venting, substance use, behavioral disengagement and self-blame. Responses were made on a 4-point Likert scale, with each 2-item subscale score ranging from 0 to 8 where a higher score represents greater use of a coping strategy. To develop coherent coping variables which reflect the data, total scores for each of the 14 COPE subscales were submitted to principle axis factor analysis using varimax rotation. The final three factor solution (not shown in this paper, available from the authors on request) included active coping ( $\alpha=.73$ ), negative coping $(\alpha=.72)$, and assisted coping $(\alpha=.85)$, and accounted for $51 \%$ of the variance. Preliminary regression analyses (not shown, available on request) assessed the contribution of demographic information collected in the questionnaire, including gender, age, ethnicity, current employment status (are you currently employed? yes/no), and parenting status (do you have children? yes/no). Significant predictors for each dependent variable

${ }^{1}$ Marital status in this study was self-defined by the respondent and included partner/ living status. It may differ from legal marital status. Divorced level is a collapse of separated and divorced. Never married level represents individuals who have never married and have not defined themselves as being in a relationship. 
in the regression analyses were entered as covariates alongside remaining marital status dummy variables. These included SRLE score, active coping and negative coping, and age (CES-D only).

\section{Data Analysis}

New variables were created as total, or subscale, scores for each questionnaire measure. A replacement by means method was used for missing responses totaling $<10 \%$ of the items for a given questionnaire. Missing data replacement was not completed for COPE scores since this measure is broken into smaller two-item subscales. Participants with missing responses $>10 \%$ for a questionnaire measure were not included in the data analysis involving that questionnaire measure. Consequently, the number of scores available varied for each measure. Due to skewed distributions, all three dependent variables were transformed using Log10.

\subsection{Moderation}

It was hypothesized that marital status might be related to psychological wellbeing, as measured by GHQ12, CES-D and SWLS score, via moderating effects of perceived level of social support. Moderation was assessed using Hayes and Matthes' (2009) MODPROBE macro in SPSS. It improves on Baron and Kenny (1986) steps by allowing the user to probe the interaction and find the specific pattern of effects of the focal predictor variable as a function of the moderator i.e. to understand the conditions under which the relationship between the focal predictor and the outcome is strong vs. weak (Hayes \& Matthes, 2009). Moderation is indicated by significant effect of $X Z$ when $X$ and $Z$ are controlled (Baron \& Kenny, 1986). Psychological wellbeing was used as the outcome variable $(Y)$, perceived social support as the potential moderating variable $(Z)$, and each marital status dummy variable was entered in turn as a predictor variable $(X)$. SRLE score, active coping and negative coping, and age (CES-D only) were entered as covariates. Transformed scores were used in the analysis due to nonnormal distribution of all three dependent variables. Values of perceived social support were set by MODPROBE macro at the sample mean and $\pm 1 S D$ from the mean. The married were used as the comparison group since it is the normative status.

\subsection{Mediation}

To assess the potential effect of third variable perceived social support on the relationship between marital status and psychological wellbeing, mediation analyses were performed using a non-parametric bootstrap macro developed by Preacher and Hayes (2008). Preacher and Hayes state that mediation can be established by testing product of paths coefficients $a$ and $b\left(a^{*} b\right)$, known as the indirect effect. The outcome variable $(Y)$ was psychological wellbeing, the predictor variable was marital status (with dummy coding), and perceived social support was entered as the potential mediating variable $(M)$. Covariates were identical to those in the moderation analyses. Preacher and Hayes' (2008) Sobel test determines the significance of the indirect effect of the mediator by testing the hypothesis of no difference between the total effect (path $c$ ) and the direct effect (path $c$ '). According to Hayes (2009), this approach is superior to the Baron and Kenny (1986) causal steps approach as it can address the significance of the indirect effects, has higher power and has lower Type I error rates. Bootstrapping is a way to overcome the limitations of statistical methods that make assumptions about normality (Preacher \& Hayes, 2008) and offers a powerful method for obtaining confidence limits for indirect effects (MacKinnon, Fairchild, \& Fritz, 2007). The analyses and bootstrap estimates that follow are based on 5000 bootstrap samples (confidence intervals set at 99\%).

\section{Results}

\subsection{Moderation}

The results provided no support for perceived social support as a moderator in the relationship between marital status and psychological wellbeing for most of the marital status groups. However, when GHQ12 was used with the cohabiting group perceived social support emerged as a significant moderator (Table 1).

Figure 1 presents the conditional effect of being in a cohabiting relationship (1) compared to all other groups (0) on GHQ12 score for high (mean $+1 S D$ ), medium (mean) and low levels (mean $-1 S D$ ) of perceived social support. Specifically, only low levels of social support predict significantly lower levels of psychological wellbeing, as measured by GHQ12 (lower confidence interval [LCI]: .01, upper confidence interval [UCI]: .20). This 
Table 1. Regression coefficients ${ }^{\mathrm{a}}$ for marital status (X), social support (Z) and interaction (XZ) on psychological wellbeing.

\begin{tabular}{ccccccccccc}
\hline & \multicolumn{3}{c}{ GHQ12 } & \multicolumn{3}{c}{ CES-D } & \multicolumn{3}{c}{ SWLS } \\
\cline { 2 - 9 } & $X$ & $Z$ & $X Z$ & $X$ & $Z$ & $X Z$ & $X$ & $Z$ & $X Z$ \\
\hline Widowed & .05 & $-.01^{* *}$ & -.01 & .25 & $-.01^{* *}$ & -.01 & .03 & $.01^{* *}$ & -.01 \\
Divorced & .08 & $-.01^{* *}$ & -.01 & -.15 & $-.01^{* *}$ & .01 & -.13 & $.01^{* *}$ & .01 \\
Cohabiting & $-.28^{*}$ & $-.01^{* *}$ & $-.01^{*}$ & .25 & $-.01^{* *}$ & -.01 & -.10 & $.01^{* *}$ & .01 \\
Never married & -.04 & $-.01^{* *}$ & .01 & .02 & $-.01^{* *}$ & -.01 & -.06 & $.01^{* *}$ & .01 \\
LAT & -.04 & $-.01^{* *}$ & .01 & -.17 & $-.01^{* *}$ & .01 & $-.27^{*}$ & $.01^{* *}$ & .01 \\
Remarried & .07 & $-.01^{* *}$ & .01 & -.17 & $-.01^{* *}$ & .01 & -.01 & $.01^{* *}$ & .01 \\
\hline
\end{tabular}

Note: a. ${ }^{*} p<.05{ }^{* *} p<.01$ Significant moderation marked in bold.

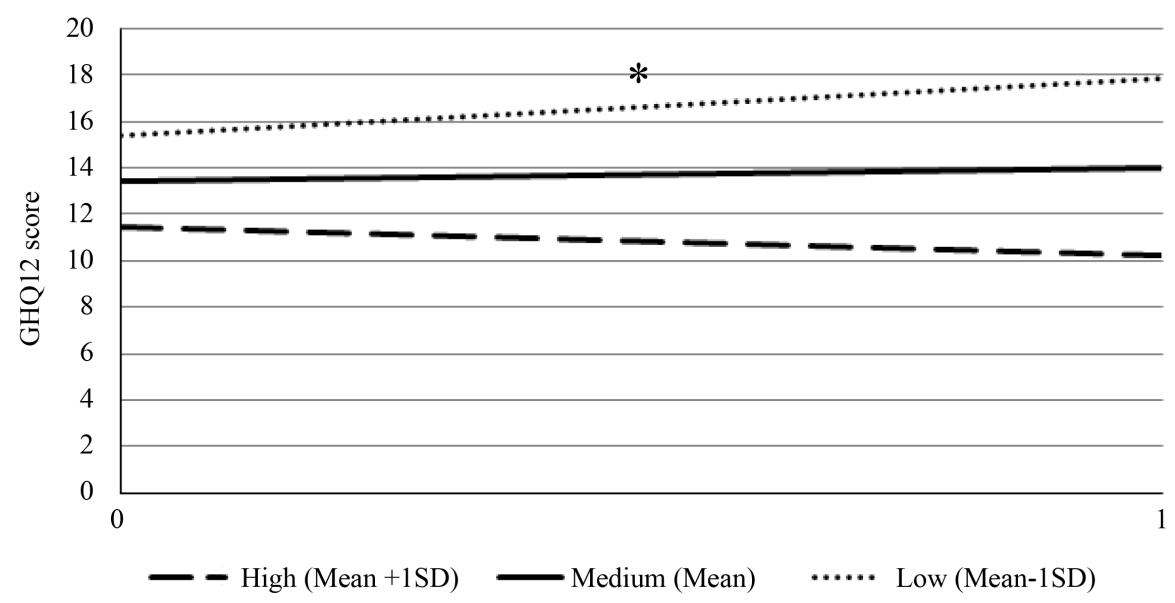

Figure 1. Conditional effect of being in a cohabiting relationship on GHQ12 at high, medium and low levels of perceived social support (MOS-SSS).

effect was not found when CES-D or SWLS were used as dependent variables, which may reflect slight differences in what aspects of psychological wellbeing are being measured in each scale.

\subsection{Mediation}

Table 2 presents the results of the mediation analysis for all models, using GHQ12, CES-D and SWLS as the dependent variable and controlling for SRLE score, active coping and negative coping, age, gender, parental status, SRLE score and COPE subscale scores. The bootstrap results indicated that the total effect of being widowed, divorced and never married was significantly reduced when perceived social support was included as a mediator. No significant mediation of perceived social support was found for the relationship between psychological wellbeing and being in a cohabiting relationship, LAT or remarried. While perceived social support was not a significant mediator in the remarried group, when GHQ12 and SWLS were entered as the dependent variables the remarried had significantly poorer levels of psychological wellbeing compared to the married. This result was not present when CES-D was entered as the dependent variable and may be a result of subtle differences between the three measures. SWLS measures global cognitive judgments of satisfaction with one's life and is distinguished from GHQ12 and CES-D in that it is more cognitively than emotionally driven (Diener et al., 1985). Patterns of mediation were generally consistent across all measures of psychological wellbeing. Total and direct effects and confidence intervals are presented in Table 2 for each of the significant mediation models using results from mediation analyses.

The bootstrap results indicated that the total effect of being widowed on psychological wellbeing, as measured by GHQ12 score (total effect $=4.72, p=.001$ ) was reduced when perceived social support was included as a mediator in the model (direct effect $=2.80, p=.010$ ). Furthermore, the analyses revealed, with $99 \%$ confidence, 
Table 2. Mediation analyses for the effect of perceived social support (M) in the association between marital status (X) and psychological wellbeing (Y).

\begin{tabular}{|c|c|c|c|c|c|c|c|c|}
\hline \multirow{2}{*}{$\begin{array}{l}\text { Dependent } \\
\text { variable }(Y)\end{array}$} & \multirow{2}{*}{$\begin{array}{l}\text { Independent } \\
\text { Variable }(X)\end{array}$} & \multirow{2}{*}{$\begin{array}{c}\text { Effect of } \\
X \text { on } M \\
\quad(a)\end{array}$} & \multirow{2}{*}{$\begin{array}{c}\text { Effect of } \\
M \text { on } Y \\
\text { (b) }\end{array}$} & \multirow{2}{*}{$\begin{array}{l}\text { Direct } \\
\text { effect } \\
\left(c^{\prime}\right)\end{array}$} & \multirow{2}{*}{$\begin{array}{l}\text { Total } \\
\text { effect } \\
(c)\end{array}$} & \multirow{2}{*}{$\begin{array}{l}\text { Indirect } \\
\text { effect } \\
(a b)\end{array}$} & \multicolumn{2}{|c|}{$99 \%$ CI } \\
\hline & & & & & & & Lower & Upper \\
\hline \multirow{6}{*}{$\begin{array}{c}\text { GHQ12 } \\
(N=501)\end{array}$} & Widowed & $-17.88^{* * *}$ & $-.11^{* * *}$ & $2.80^{* *}$ & $4.72^{* * *}$ & $1.92^{* *}$ & 1.1232 & 2.9527 \\
\hline & Divorced & $-12.82^{* * *}$ & $-.11^{* * *}$ & .50 & $1.88^{*}$ & $1.38^{* *}$ & .7308 & 2.1643 \\
\hline & Cohabiting & 2.82 & $-.11^{* * *}$ & -.10 & -.40 & -.30 & -.7230 & .0679 \\
\hline & Never Married & $-7.60^{* * *}$ & $-.11^{* * *}$ & .21 & 1.02 & $.82^{* *}$ & .3593 & 1.4986 \\
\hline & LAT & 4.17 & $-.11^{* * *}$ & .45 & .01 & -.45 & -.9531 & .0415 \\
\hline & Remarried & -3.46 & $-.11^{* * *}$ & 1.73 & 1.35 & .37 & -.1230 & 1.0109 \\
\hline \multirow{6}{*}{$\begin{array}{c}\text { CES-D } \\
(N=500)\end{array}$} & Widowed & $-16.19^{* * *}$ & $-.21^{* * *}$ & $5.76^{* *}$ & $9.19^{* * *}$ & $3.42^{* *}$ & 2.0573 & 5.1260 \\
\hline & Divorced & $-11.60^{* * *}$ & $-.21^{* * *}$ & 1.05 & $3.50^{*}$ & $2.44^{* *}$ & 1.3741 & 3.9328 \\
\hline & Cohabiting & .72 & $-.21^{* * *}$ & -.52 & -.68 & -.14 & -.9392 & .6818 \\
\hline & Never Married & $-9.80^{* * *}$ & $-.21^{* * *}$ & -.79 & 1.28 & $2.09^{* *}$ & 1.0767 & 3.4486 \\
\hline & LAT & 1.75 & $-.21^{* * *}$ & .55 & .18 & -.38 & -1.2801 & .5006 \\
\hline & Remarried & -2.62 & $-.21^{* * *}$ & .99 & 1.54 & .56 & -.4752 & 1.7025 \\
\hline \multirow{6}{*}{$\begin{array}{c}\text { SWLS } \\
(N=500)\end{array}$} & Widowed & $-17.88^{* * *}$ & $.14^{* * *}$ & $-4.57^{* * *}$ & $-7.01^{* * *}$ & $-2.43^{* *}$ & -3.5403 & -1.5596 \\
\hline & Divorced & $-12.86^{* * *}$ & $.14^{* * *}$ & $-2.50^{*}$ & $-4.26^{* * *}$ & $-1.76^{* *}$ & -2.6520 & -1.0155 \\
\hline & Cohabiting & 2.82 & $.14^{* * *}$ & -.23 & .15 & .38 & -.0623 & .9275 \\
\hline & Never Married & $-7.60^{* * *}$ & $.14^{* * *}$ & -1.53 & $-2.56^{*}$ & $-1.05^{* *}$ & -1.7733 & -.4602 \\
\hline & LAT & 4.17 & $.14^{* * *}$ & $-1.99^{*}$ & -1.42 & .57 & -.0518 & 1.1748 \\
\hline & Remarried & -3.46 & $.14^{* * *}$ & $-1.93^{*}$ & $-2.40^{* *}$ & -.47 & -1.1842 & .1659 \\
\hline
\end{tabular}

that the total indirect effect (i.e. the difference between the total and direct effects) of being widowed on psychological wellbeing through perceived social support was significant, with a point estimate of 1.92 and a 99\% bias-corrected (BC) bootstrap confidence interval of 1.12 (lower: LCI) to 2.95 (upper: UCI). Similar results were found when CES-D and SWLS were used as the predictor variable (path coefficients in Table 2). Being widowed, therefore, exerts an indirect effect on psychological wellbeing through lower levels of perceived social support. Figure 2 illustrates the partial mediation of perceived social support in the relationship between being widowed and psychological wellbeing.

The total effect of being divorced on psychological wellbeing, as measured by GHQ12 score (total effect = $1.88, p=.048$ ) became non-significant when perceived social support was included as a mediator in the model $($ direct effect $=.50, p=.629)$, with a significant indirect effect (point estimate $=1.38, \mathrm{LCI}=.73 \mathrm{UCI}=2.16$ ). Results revealed that, compared to the married, divorced individuals had significantly lower levels of psychological wellbeing through lower levels of perceived social support. As the total effect was reduced to being non-significant, perceived social support appears to fully mediate the relationship between being divorced and psychological wellbeing. Similar results were found when CES-D and SWLS were used as the predictor variable. For path coefficients please refer to Table 2 and Figure 3.

The bootstrap results indicated that the total effect of being never married on psychological wellbeing, as measured by GHQ12 score (total effect $=1.20, p=.191$ ) was reduced when perceived social support was included as a mediator in the model (direct effect $=.21, p=.788$ ), with a significant indirect effect (point estimate $=.82$, LCI $=.36 \mathrm{UCI}=1.50$ ). The never married were not significantly more distressed than the married. However, it is possible for $M$ (perceived social support) to be causally related between $X$ (marital status) and $Y$ (psychological wellbeing), even if $X$ and $Y$ are not significantly associated (Hayes, 2009). Being never married, therefore, exerts a significant indirect effect on psychological wellbeing through lower levels of perceived social support (Table 2 and Figure 4). When SWLS was used as the predictor variable, the never married reported 


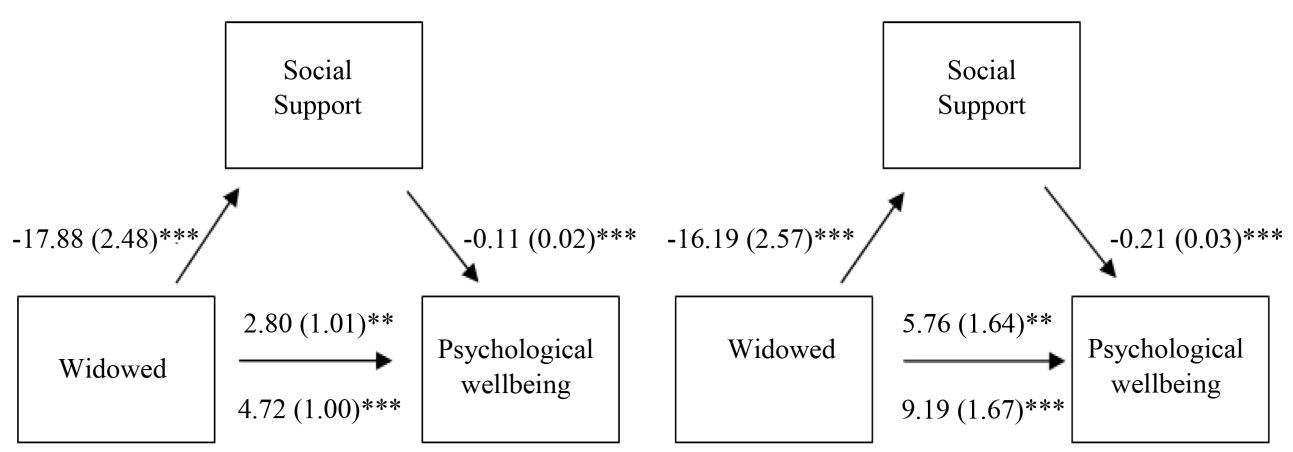

(a)

(b)

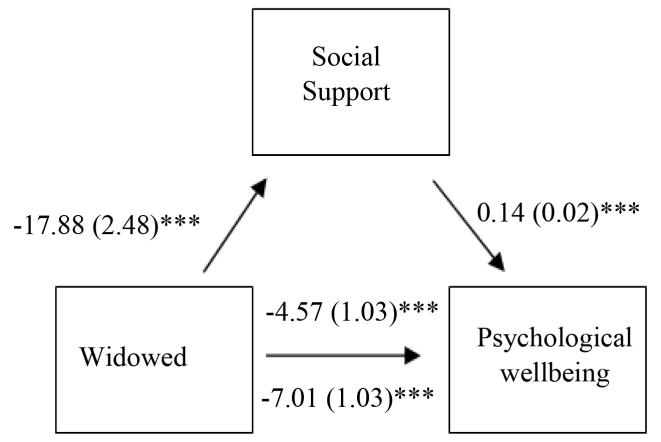

(c)

Figure 2. Mediation model where being widowed exerts an indirect effect on psychological wellbeing (a) GHQ12; (b) CES-D; (c) SWLS through perceived social support (MOS-SSS).

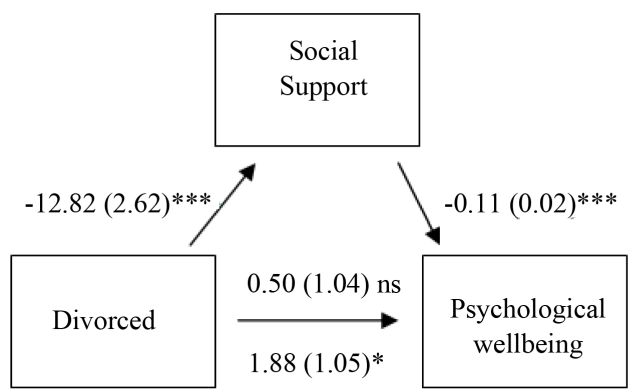

(a)

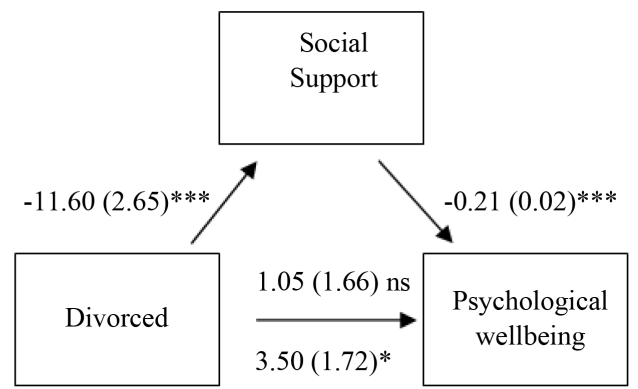

(b)

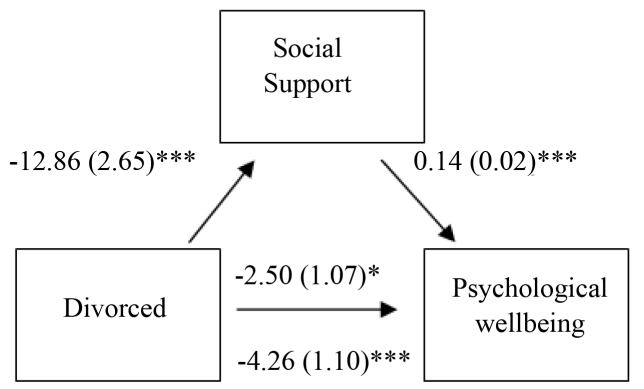

(c)

Figure 3. Mediation model where being divorced exerts an indirect effect on psychological wellbeing (a) GHQ12; (b) CES-D; (c) SWLS through perceived social support (MOS-SSS).

significantly lower levels of psychological wellbeing compared to the married (total effect $=-2.56 p=.002$ ). The never married are likely to represent a heterogeneous group including those who are self-defined single and yet to marry as well as those who do not intend to marry. Thus, it is expected that this difference in SWLS is due 


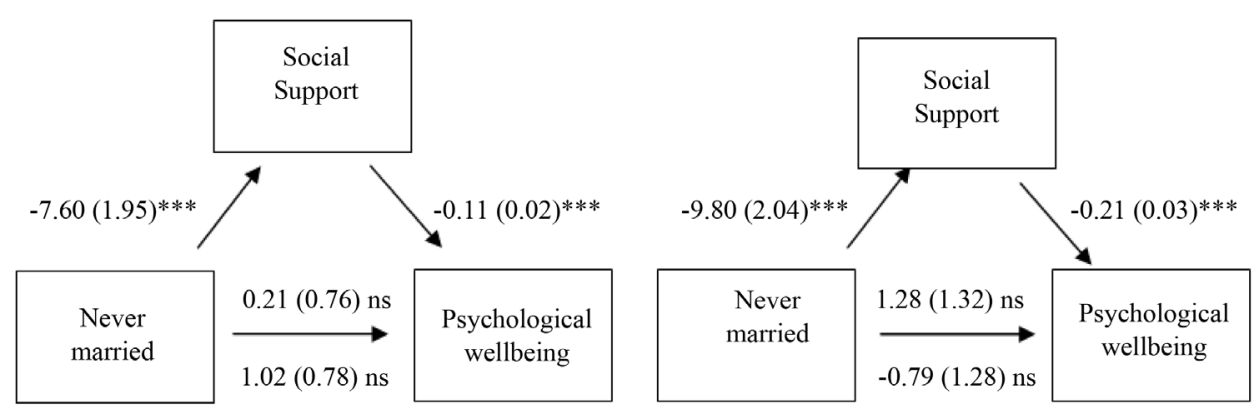

(a)

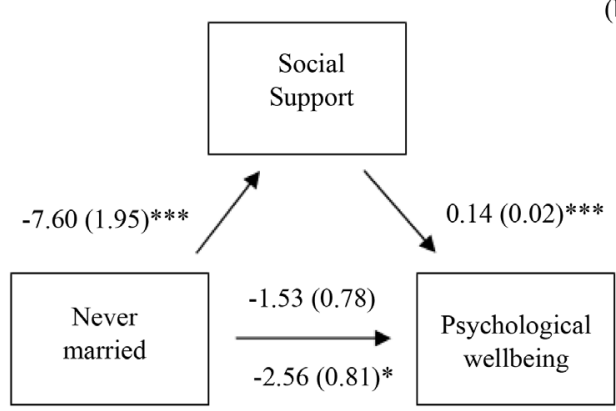

(b)

(c)

Figure 4. Mediation model where being never married exerts an indirect effect on psychological wellbeing (a) GHQ12; (b) CES-D; (c) SWLS through perceived social support (MOS-SSS).

to age related differences in life satisfaction where being older and never married may have a negative influence on life review, as measured by SWLS. When perceived social support was controlled for, the difference became non-significant (direct effect $=-1.53, p=.052$ ), indicating significant mediation of social support (point estimate $=-1.05$, LCI $=-1.77 \mathrm{UCI}=-.46)$.

\section{Discussion}

This study used cross-sectional questionnaire data to evaluate the role of perceived social support in the relationship between marital status and psychological wellbeing. First, perceived social support was found to have a direct, health promoting effect on psychological health across all three measures, independent of marital status. Marital status was also associated with psychological health where the widowed and divorced scored themselves significantly lower than the continuously married on all three measures of psychological health; never married and remarried participants reported significantly lower levels of life satisfaction relative to the married; and levels of psychological wellbeing in the cohabiting and LAT groups were found to be similar to that of the married. Further, marital status differences emerged in perceived social support and the widowed, divorced and never married reported significantly lower levels relative to the married. These results corroborate previous findings of a relationship between perceived social support and psychological wellbeing (Thoits, 1995; Wyke \& Ford, 1992); a relationship between marital status and psychological wellbeing (Wyke \& Ford, 1992; Waldron, Hughes, \& Brooks, 1996); and of marital status differences in perceived social support (Duffy, 1993; Ross, 1995).

Finally, using moderation and mediation analyses, the results add to the existing literature by highlighting perceived social support as a key mechanism through which marital status affects psychological health (Barrett, 1999; Hewitt et al., 2010). There was little evidence of moderation effects of social support in the current data set, except for the cohabiting group where low social support leads to poorer psychological health when measured using GHQ12. However, bootstrap mediation analyses indicate that, with the married as the comparison group, the psychological wellbeing of the widowed, the divorced and the never married is significantly mediated by perceived social support, even when other important variables including number of daily hassles and coping strategies are held constant. This pattern of findings also provides evidence for a direct relationship between psychological wellbeing and perceived social support, where higher levels of perceived social support allows for better levels of psychological wellbeing. Interestingly, perceived social support accounts for lower levels of 
psychological wellbeing in the divorced compared to the married more than was the case in the widowed group, where perceived social support only partially mediated the relationship between being widowed and psychological wellbeing, and in the never married group, where only the indirect effect was significant. This indicates that in widowhood, but not necessarily in divorce, there may be other key factors, such as the circumstances of the loss, which were not measured in this study that explain the significantly lower levels of psychological wellbeing relative to the married.

In the existing marital status literature, protection hypotheses suggest a health protective benefit of marriage (Waldron et al., 1996). However, contrary to marital protection, the findings reveal that the never married, as well as those in cohabiting and LAT relationships, report similar levels of psychological wellbeing to their married counterparts. Perceived social support is clearly an important resource that has a significant impact on psychological wellbeing. However, the findings suggest that its benefits are not unique to married relationships. The cohabiters and LATs are not in a married relationship with access to all of the benefits conferred by being married, yet show the same levels of psychological wellbeing, providing support for the notion that the presence of a supportive spouse or partner may be more important for psychological health than marital status per se (Dush \& Amato, 2005; Ross, 1995; Soons \& Liefbroer, 2008).

This investigation has both strengths and limitations. There has been increasing interest in the relationship between perceived social support and psychological wellbeing and there is a large existing body of literature that considers the differences in psychological wellbeing between marital status groups. However, to date, there have been few systematic analyses of the relationship between these three variables. Seeking to evaluate these relationships, this study made use of a large sample of men and women and the depth of the study methodology allowed for conclusions to be drawn about different marital and relationship status groups, rather than simply grouping people into married and unmarried categories. In addition, by employing three different measures of psychological wellbeing the investigation was able to consider psychological distress as well as depressive symptoms and life satisfaction. Patterns of mediation are similar across all measures, suggesting that the mediating effect of perceived social support is important for different aspects of psychological wellbeing.

However, the study sample is relatively small and the generalizability of the findings of this study may be limited by the study's use of opportunity sampling. Further, the results of these analyses are based on crosssectional data and so may not accurately reflect longitudinal mediation effects. The analyses cannot control for differences in baseline psychological wellbeing in those who marry or enter into relationships, compared with those who do not, nor is there any control for how long people have been in their current marital status. Changes in participants' perceptions of social support over time are also missing from these analyses. Thus, some caution must be exercised in making definitive interpretations of these results. Larger scale longitudinal data analysis would allow for the investigation of causal pathways and to evaluate possible selection effects as possible explanations for the findings. In addition, it is important to acknowledge that there could be other competing variables that could also play a mediating role in the relationship between marital status and psychological wellbeing that are not measured here. This study evaluated general perceived social support scores. However, future investigations may examine the multiple dimensions and sources of social support, and their effects on the relationship between marital status and psychological wellbeing. In addition, it would be interesting to consider the role of social interaction in the relationship between marital status and wellbeing, since the patterns may mirror those for perceived social support.

\section{Conclusion}

This study makes a valuable contribution to the understanding of the relationship between marriage and psychological health. Both perceived social support and marital status were independently associated with psychological wellbeing. Moreover, perceived social support emerged as a significant mediator in the relationship between marital status and psychological wellbeing. Specifically, this investigation found that perceived social support accounts for marital status differences in psychological health and that lower levels of perceived social support in the widowed, divorced and never married, relative to the married, result in significantly poorer psychological health. This study highlights the importance of perceived social support for psychological wellbeing, particularly for people who are not in a relationship, namely the widowed, divorced and never married, and suggests that marriage itself is not the key to better wellbeing. Rather, it is the social support afforded by being in a relationship and being embedded in a secure social network that promotes psychological health. 


\section{References}

Baron, R. M., \& Kenny, D. A. (1986). The Moderator-Mediator Variable Distinction in Social Psychological Research: Conceptual, Strategic, and Statistical Considerations. Journal of Personality \& Social Psychology, 51, 1173-1182. http://dx.doi.org/10.1037/0022-3514.51.6.1173

Barrett, A. E. (1999). Social Support and Life Satisfaction among the Never Married: Examining the Effects of Age. Research on Aging, 21, 46-72. http://dx.doi.org/10.1177/0164027599211003

Bierman, A., Fazio, E. M., \& Milkie, M. A. (2006). A Multifaceted Approach to the Mental Health Advantage of the Married. Journal of Family Issues, 27, 554-582. http://dx.doi.org/10.1177/0192513X05284111

Carver, C. S. (1997). You Want to Measure Coping but Your Protocol’s Too Long: Consider the Brief COPE. International Journal of Behavioral Medicine, 4, 92-100. http://dx.doi.org/10.1207/s15327558ijbm0401_6

Cohen, J., \& Cohen, P. (1983). Applied Multiple Regression/Correlation Analysis for the Behavioral Sciences. Hillsdale, NJ: Erlbaum.

Cohen, S., \& Wills, T. A. (1985). Stress, Social Support, and the Buffering Hypothesis. Psychological Bulletin, 98, $310-357$. http://dx.doi.org/10.1037/0033-2909.98.2.310

Coyne, J. C., \& DeLongis, A. (1986). Going beyond Social Support: The Role of Social Relations in Adaptation. Journal of Consulting \& Clinical Psychology, 54, 454-460. http://dx.doi.org/10.1037/0022-006X.54.4.454

Diener, E., Emmons, R. A., Larsen, R. J., \& Griffin, S. (1985). The Satisfaction with Life Scale. Journal of Personality Assessment, 49, 71-75. http://dx.doi.org/10.1207/s15327752jpa4901_13

Duffy, M. E. (1993). Social Networks and Social Support of Recently Divorced Women. Public Health Nursing, 10, 19-24. http://dx.doi.org/10.1111/j.1525-1446.1993.tb00015.x

Dush, C. M. K., \& Amato, P. R. (2005). Consequences of Relationship Status and Quality for Subjective Well-Being. Journal of Social \& Personal Relationships, 22, 607-627. http://dx.doi.org/10.1177/0265407505056438

Goldberg, D. (1978). Manual of the GHQ. Windsor, UK: NFER-Nelson.

Gove, W. R., Hughes, M., \& Style, C. B. (1983). Does Marriage Have Positive Effects on the Psychological Well-Being of the Individual? Journal of Health \& Social Behavior, 24, 122-131. http://dx.doi.org/10.2307/2136639

Hayes, A. F. (2009). Beyond Baron and Kenny: Statistical Mediation Analysis in the New Millennium. Communication Monographs, 76, 408-420. http://dx.doi.org/10.1080/03637750903310360

Hayes, A. F., \& Matthes, J. (2009). Computational Procedures for Probing Interactions in OLS and Logistic Regression: SPSS and SAS Implementations. Behavior Research Methods, 41, 924-936. http://dx.doi.org/10.3758/BRM.41.3.924

Hewitt, B., Turrell, G., \& Giskes, K. (2010). Marital Loss, Mental Health and the Role of Perceived Social Support: Findings from Six Waves of an Australian Population Panel Study. Journal of Epidemiology \& Community Health, 66, 308-314. http://dx.doi.org/10.1136/jech.2009.104893

House, J. S., Landis, K. R., \& Umberson, D. (1988). Social Relationships and Health. Science, 241, 540-545. http://dx.doi.org/10.1126/science.3399889

Kohn, P. \& Macdonald, J. E. (1992). The Survey of Life Experiences: A Decontaminated Hassles Scale for Adults. Journal of Behavioral Medicine, 15, 221-236. http://dx.doi.org/10.1007/BF00848327

Krantz, J. H., \& Dalal, R. (2000). Validity of Web-Based Psychological Research. In M. H. Birnbaum (Ed.), Psychological Experiments on the Internet (pp. 35-60). San Diego, CA: Academic Press. http://dx.doi.org/10.1016/B978-012099980-4/50003-4

Marks, N. F. (1996). Flying Solo at Midlife: Gender, Marital Status, and Psychological Well-Being. Journal of Marriage \& Family, 58, 917-932. http://dx.doi.org/10.2307/353980

MacKinnon, D. P., Fairchild, A. J., \& Fritz, M. S. (2007). Mediation Analysis. Annual Reviews of Psychology, 58, 593-614. http://dx.doi.org/10.1146/annurev.psych.58.110405.085542

McLaughlin, J., Horwitz, A. V., \& White, H. R. (2002). The Differential Importance of Friend, Relative and Partner Relationships for the Mental Health of Young Adults. Advances in Medical Sociology, 8, 223-246. http://dx.doi.org/10.1016/S1057-6290(02)80028-6

Overbeek, G., Vollebergh, W., de Graaf, R., Scholte, R., de Kemp, R., \& Engels, R. (2006). Longitudinal Associations of Marital Quality and Marital Dissolution with the Incidence of DSM-III-R Disorders. Journal of Family Psychology, 20, 284-291. http://dx.doi.org/10.1037/0893-3200.20.2.284

Pavot, W., \& Diener, E. (1993). Review of the Satisfaction with Life Scale. Psychological Assessment, 5, 164-172. http://dx.doi.org/10.1037/1040-3590.5.2.164

Pearlin, L. I., \& Johnson, J. S. (1977). Marital Status, Life-Strains and Depression. American Sociological Review, 42, 704715. http://dx.doi.org/10.2307/2094860 
Preacher, K. J., \& Hayes, A. F. (2008). Asymptotic and Resampling Strategies for Assessing and Comparing Indirect Effects in Multiple Mediator Models. Behavior Research Methods, 40, 879-891. http://dx.doi.org/10.3758/BRM.40.3.879

Pugliesi, K., \& Shook, S. L. (1998). Gender, Ethnicity and Network Characteristics: Variation in Social Support Resources 1. Sex Roles, 38, 215-238. http://dx.doi.org/10.1023/A:1018733116398

Radloff, L. S. (1977). The CES-D Scale: A Self-Report Depression Scale for Research in the General Population. Applied Psychological Measurement, 1, 385-401. http://dx.doi.org/10.1177/014662167700100306

Reips, U. D. (2002). Standards for Internet-Based Experimenting. Experimental Psychology, 49, 243-256. http://dx.doi.org/10.1026//1618-3169.49.4.243

Ross, C. E. (1995). Reconceptualizing Marital Status as a Continuum of Social Attachment. Journal of Marriage \& Family, 57, 129-140. http://dx.doi.org/10.2307/353822

Schmidt, W. (1997). World-Wide Web Survey Research: Benefits, Potential Problems and Solutions. Behavior Research Methods, Instruments, \& Computers, 29, 274-279.

Sherbourne, C. D., \& Hays, R. D. (1990). Marital Status, Social Support and Health Transitions in Chronic Disease Patients. Journal of Health \& Social Behavior, 31, 328-342. http://dx.doi.org/10.2307/2136817

Sherbourne, C. D., \& Stewart, A. L. (1991). The MOS Social Support Survey. Social Science \& Medicine, 32, 705-714. http://dx.doi.org/10.1016/0277-9536(91)90150-B

Soons, J. P. M., \& Liefbroer, A. C. (2008). Together Is Better? Effects of Relationship Status and Resources on Young Adults’ Well-Being. Journal of Social \& Personal Relationships, 2, 603-624. http://dx.doi.org/10.1177/0265407508093789

Stutzer, A., \& Frey, B. S. (2006). Does Marriage Make People Happy, or Do Happy People Get Married? Journal of Socio-Economics, 35, 326-347. http://dx.doi.org/10.1016/j.socec.2005.11.043

Thoits, P. (1995). Stress, Coping and Social Support Processes: What Are We? What Next? Journal of Health \& Social Behavior, 35, 53-79. http://dx.doi.org/10.2307/2626957

Waite, L. J. (1995). Does Marriage Matter? Demography, 32, 483-507. http://dx.doi.org/10.2307/2061670

Waite, L. J., \& Gallagher, M. (2000). The Case for Marriage: Why Married People Are Happier, Healthier and Better off Financially. New York: Doubleday.

Waldron, I., Hughes, M. E., \& Brooks, T. L. (1996). Marriage Protection and Marriage Selection: Prospective Evidence for Reciprocal Effects of Marital Status and Health. Social Science \& Medicine, 43, 113-123. http://dx.doi.org/10.1016/0277-9536(95)00347-9

Walker, K. N., MacBride, A., \& Vachon, M. L. S. (1977). Social Support Networks and the Crisis of Bereavement. Social Science \& Medicine, 11, 35-41. http://dx.doi.org/10.1016/0037-7856(77)90143-3

Wyke, S., \& Ford, G. (1992). Competing Explanations for Associations between Marital Status and Health. Social Science \& Medicine, 34, 523-532. http://dx.doi.org/10.1016/0277-9536(92)90208-8 